\title{
Segmentation of Meningiomas and Low Grade Gliomas in MRI
}

\author{
M. R. Kaus ${ }^{1,3}$, S. K. Warfield ${ }^{1}$, A. Nabavi ${ }^{1,2}$, E. Chatzidakis ${ }^{1,2}$, P. M. Black ${ }^{2}$, \\ F. A. Jolesz ${ }^{1}$, and R. Kikinis ${ }^{1}$ \\ 1 Surgical Planning Laboratory, Department of Radiology \\ 2 Department of Neurosurger \\ Brigham and Women's Hospital \\ Harvard Medical School, 75 Francis St., Boston, MA 02115 \\ 3 Lehrstuhl Technische Elektronik \\ Universität Erlangen-Nürnberg, D-91058 Erlangen, Germany \\ \{kaus, warfield, arya, manos, jolesz, kikinis\}@bwh.harvard.edu \\ pmblack@bics. bwh. harvard.edu \\ http://splweb.bwh.harvard.edu:8000
}

\begin{abstract}
Computer assisted surgical planning and image guided technology have become increasingly used in neurosurgery. We have developed a system based on ATmC (Adaptive Template moderated Classification) for the automated segmentation of 3D MRI brain data sets of patients with brain tumors (meningiomas and low grade gliomas) into the skin, the brain, the ventricles and the tumor. In a validation study of 13 patients with brain tumors, the segmentation results of the automated method are compared to manual segmentations carried out by 4 independent trained human observers. It is shown that the automated method segments brain and tumor with accuracy comparable to the manual method and with improved reproducibility.
\end{abstract}

Keywords: Surgical planning, Image guided neurosurgery, Magnetic resonance $(\mathrm{MR})$, segmentation, registration, brain, tumor

\section{Introduction}

Computer assisted surgical planning and image guided technology have become increasingly used in neurosurgery $[1,9,15,21]$. 2D images accurately describe the size and location of anatomical objects. The process of generating 3D views to highlight structural information and spatial relationships of the anatomy, however, is a difficult task and usually carried out in the clinician's mind. Image processing tools can provide the surgeon with interactively displayed 3D visual information to facilitate the comprehension of the entire anatomy, and improve the spatial information about relationships of critical structures (e.g. motory and sensory cortex, vascular structures) and pathology [12].

Today commercially available systems usually provide the surgeon only with $2 \mathrm{D}$ cross-sections of the intensity value images and a $3 \mathrm{D}$ model of the 
skin. The main limiting factor for the routine use of 3D models of other important structures in clinical practice is the amount of time that an operator has to spend in the preparation of the data $[7,14]$. The availability of automated methods will significantly reduce the time and is necessary to make such methods practical.

Conventional segmentation methods for tumor segmentation such as statistical classification or mathematical morphological operations may work well in some cases but may not differentiate between enhancing tumor, edema and normal tissue $[8,17,18]$. For the separation of these tissues, the acquisition of several tissue parameters alone has been shown to be insufficient [10]. A combination of statistical classification and anatomical information has been used for the segmentation of MRI images of the brain [3,11,20]. In a recent study, an anatomical knowledge guided fuzzy c-means method was used for automatic detection and segmentation of glioblastoma multiforme from a combination of T1-, T2- and Proton density (PD) MR images with promising results [2].

We have developed an automated segmentation method based on ATmC (Adaptive Template moderated Classification) [19] that combines statistical classification with anatomical knowledge from a digital atlas. The algorithm segments the skin surface, the brain, the ventricles and some of the most common tumor types, meningiomas and low grade gliomas. The purpose of the current study was to assess the accuracy and robustness of the algorithm by comparing the automated method to manual segmentation carried out by trained medical experts.

\section{Materials and Methods}

\subsection{Patient Image Data}

The MRI datasets consisted of a 3D sagittal spoiled gradient recalled (SPGR) acquisition (field of view (FOV): $240 \mathrm{~mm}$; slice-thickness: $1.5 \mathrm{~mm}$; $256 \times 256 \times 124$ matrix) after gadolinium-enhancement. 13 different patients with brain tumors of different size, shape and location were selected, i.e. 5 meningiomas (cases No. 1-3, 11, 12), and 8 low grade gliomas (cases No. 4-10, 13). A development database (cases No. 1-10) used for the design and validation of the automated segmentation method was extracted from a neurosurgical image database of approximately. 100 brain tumor cases that had been post-processed for image guided neurosurgery (manual outlining of the structures skin-surface, brain, ventricles, vessels and tumor). These cases provided a representative selection of meningioma and low grade glioma cases. Validation was also carried out on the datasets of 3 patients (cases No. 11-13) were image acquisition and processing took place after completion of the algorithm development.

\subsection{Automated Segmentation of Brain and Tumor}

We adapted a general algorithm intended for the automated segmentation of anatomical objects in different locations in the human body [19]. The algorithm 


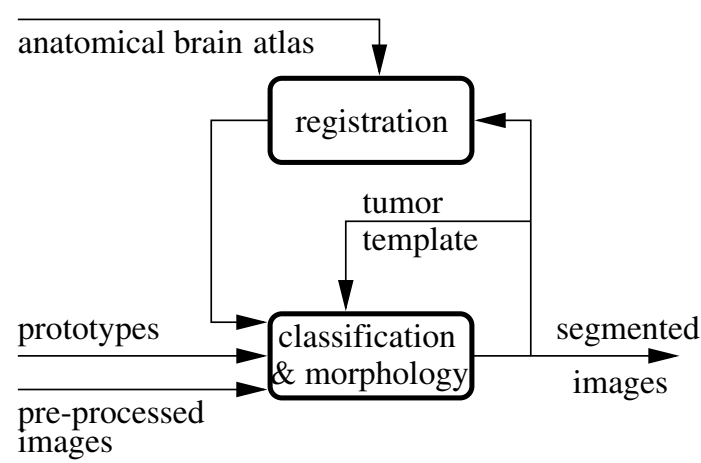

(a)

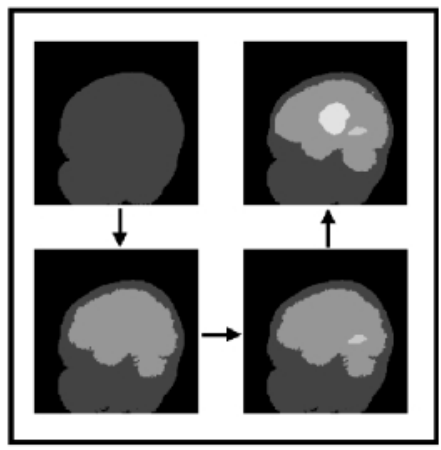

(b)

Fig. 1. ATmC (Adaptive Template moderated Classification) segmentation scheme (a) and brain tumor segmentation flow diagram (b).

combines two approaches to image segmentation into an iterative process: statistical classification and segmentation by registration of an anatomical atlas (Fig. 1). We summarize here the concept of the segmentation framework and its application to brain tumor segmentation. For a mathematical description we refer to [19].

Image Segmentation Statistical classification (k-Nearest Neighbor rule) divides the image into different tissue classes based on the signal intensity value [5]. Overlap between signal intensity distributions of different tissue classes leads to mis-classifications. To resolve this problem, additional information is derived from a digital volumetric atlas of a normal brain that has been manually segmented into approximately. 250 different structures by medical experts [12]. By projecting anatomical templates from the atlas onto the individual patient data, different structures of interest in the patient dataset can be located according to their location in the atlas.

Comparing the images of two different brains requires non-linear registration for the projection of the atlas onto the patient data, capturing individual differences by allowing structures to shrink, grow, and twist, and to move or rotate locally and independently [4]. In our approach, the algorithm computes the spatial nonlinear transform on the basis of the segmented images, rather than the original signal intensity values, in order that the registration be less susceptible to image noise and intensity artifacts.

Instead of directly projecting anatomical templates onto the patient and thus having to rely on hard boundaries, a model of anatomical localization was formulated that reflects lower confidence in the localization towards the boundary of structures ("soft boundaries"). This was implemented by using Euclidian distance transforms computed from the templates as additional anatomical feature 
channels in the kNN classification. The approach has the advantage that a very precise registration is not necessary, because the method uses both the MR intensity information and the soft spatial location.

Statistical classification and registration of the anatomical brain atlas are iterated. The goal of the iteration is to improve the result of the registration by providing tentative image segmentations, and to improve the result of the classification by providing regions of interest.

Objects of interest are defined on the classified images, where every voxel was labeled according to the assigned tissue class. For the identification of each structure and removement of classification artifacts, a local segmentation strategy was used, consisting of a) a morphological erosion to "cut" classification artifacts such as thin connections between different objects, b) a connectedcomponent algorithm to re-label every voxel as belonging to one object or another and c) a morphological dilation to restore previously eroded voxels on the object boundaries [16].

Application to Tumor Segmentation Five tissue classes were modeled: background, skin (fat/bone), brain, ventricles, and tumor. Due to the homogeneous tissue composition of meningiomas and low grade gliomas one tissue class was sufficient for the statistical model. A simple, hierarchical model of anatomy was used to define the order in which the different structures were segmented. By proceeding hierarchically from the outside to the inside of the head (Fig. 1), each segmented structure provided additional anatomical knowledge (i.e. a refined region of interest) for the next structure to be segmented. A standard normal brain atlas contains no tumor template. This has three consequences. First, anatomical templates from the atlas were derived only for the head, the ICC and the ventricles. Second, because the registration paradigm assumes correspondence between every structure in atlas and patient, a compound tissue class of the normal and pathologic brain structures was formed during ICC registration. The atlas brain was registered to the patient brain and pathology. Third, in a first tumor segmentation iteration, only atlas brain and ventricle templates were used. In a second iteration, the tumor segmentation from the first iteration was used as an anatomical template. Although this template was approximate, the additional information about the location of the tumor prevented the mis-classification of brain.

Initialization of the Automated Segmentation Method Prior to the segmentation, the image data is preprocessed with an anisotropic diffusion filtering method to reduce the noise in the MR images while preserving edges [6]. The method requires the selection of 3-4 example points for each tissue class. For the $2 \mathrm{D}$ display of MR slices and the selection of example tissue points using a mouse a graphical user interface was developed. The program calculated a statistical model for the distribution of the grey values based on these manually selected tissue prototypes. 


\subsection{Validation}

Since there is no "gold standard" to compare with, our definition of a segmentation "gold standard" is based upon the opinion of the medical expert, manifested in manual segmentations using interactive computer segmentation tools. However, manual segmentation is subject to inter-rater variability and human error. To minimize the influence of these factors while maintaining the means of measuring the segmentation accuracy of the individual raters, the standard was defined as the area of those voxels where at least 3 out of 4 experts agreed upon the identification. To determine inter- and intra-variability of the segmentation results, a fifth rater manually segmented each selected 2D slice 4 times over a period of one week, and the 4 experts carried out repeated initialization of the automated algorithm.

The experimental setup was the following: The automated algorithm was trained on a single MR slice containing the structures of interest and executed, resulting in a segmentation of the entire $3 \mathrm{D}$ dataset into the structures skin, brain, ventricles and tumor. A single $2 \mathrm{D}$ slice was randomly selected from the subset of MR slices containing the tumor. On those slices, brain and tumor were manually segmented by 4 trained medical experts using an interactive segmentation tool (MRX, GE Medical Systems, Schenectady, NY). The structures were outlined slice-by-slice by pointing and clicking with a mouse. The program connected consecutive points with lines. An anatomical object was defined by a closed contour, and the program labeled every voxel of the enclosed volume.

Statistical analysis was carried out by comparing the volumes of the automatically with the manually segmented structures. Accuracy was defined as the percentage of correctly classified voxels with respect to the total number of voxels in the image. To measure the inter- and intra-rater variation, the coefficient of variation $(\mathrm{CV} \%=100 *[(\mathrm{SD}$ volume $) /($ mean volume $)], \mathrm{SD}$ : standard deviation $)$ of the volume of the structure was calculated.

\section{Results and Discussion}

Examples for manual and automated segmentation (Fig. 2) for a meningioma (top row) and a low grade glioma (bottom row) illustrate high similarity between the two methods. Fig. 3 shows the accuracy for brain and tumor segmentation achieved by the automated and the manual method. The segmentation accuracy of the cases $11-13$ is displayed in Tab. 1. The segmentation accuracy with the automated method is above $95 \%$ for brain and above $99 \%$ for tumor, and within or close (maximum difference $0.6 \%$ ) to the range of the minimum and maximum of the accuracy with the manual method. The errors of the automated brain segmentation are in part due to the over- and under-segmentation in the area of the tentorium cerebelli and the area of the lateral sulcus with abundant vessels. The algorithm tends to oversegment these areas, if voxels e.g. of the neck close to the cerebellum are mis-classified as brain and the template ICC derived from the atlas is mis-registered. 


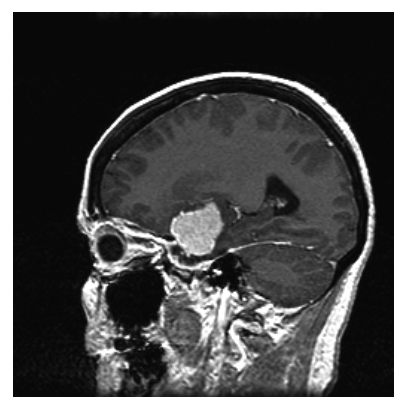

(a)

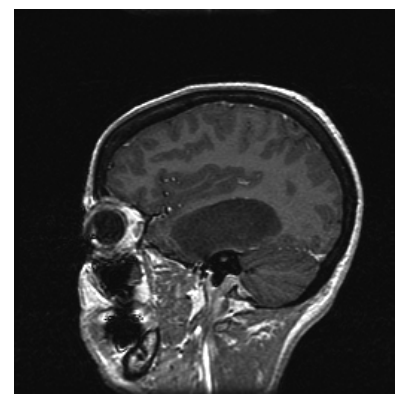

(d)

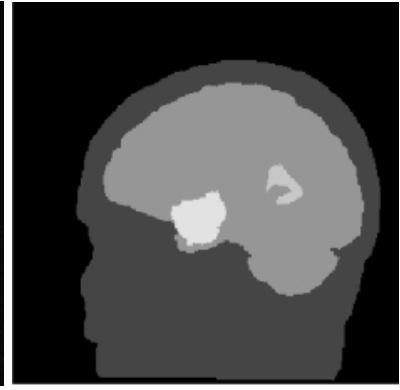

(b)

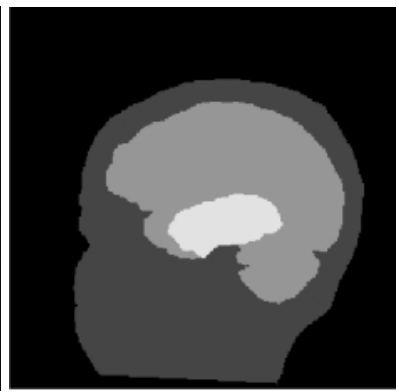

(e)

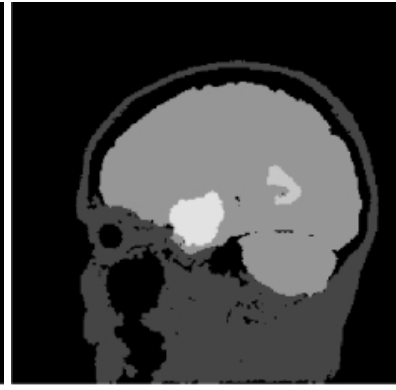

(c)

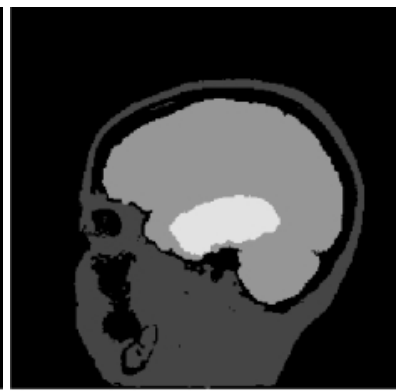

(f)

Fig. 2. Examples of manual and automated segmentation: Meningioma (SPGR image (a), manual (b), and automated segmentation (c)). Low Grade Glioma (SPGR image (d), manual (e), and automated segmentation (f).

The size of the structure affects the segmentation accuracy. Potentially, the boundaries are the areas of segmentation error. Since the comparison is based on measuring the number of correctly classified voxels (fore- and background), large objects tend to have a lower accuracy since there are more boundary voxels to mis-classify with respect to the entire image.

Fig. 4 and Fig. 5 show the inter- and intra-rater variability achieved by the manual and the automated methods. The horizontal lines mark the mean coefficient of variability over all 10 cases.

The inter- and intra-observer variability of both methods are lower for the brain than for the tumor. This is because the methods are consistent in labeling the "center" of an object, but vary in the determination of the boundaries. Since the brain is a larger structure than the tumor, the disagreement on the brain boundary with respect to the overall brain volume (not the entire image, as for the accuracy measurement) is less significant than for the tumor. 
Table 1. Segmentation accuracy of the three cases 11-13, where image data was acquired and segmented after completion of the algorithm development.

\begin{tabular}{|c|c|c|c|c|c|c|c|}
\hline \multirow{3}{*}{ Tumor Histology } & \multicolumn{3}{|c|}{ Brain Accuracy [\%] } & \multicolumn{4}{|c|}{ Tumor Accuracy [\%] } \\
\hline & \multicolumn{2}{|c|}{ Manual } & \multirow{2}{*}{$\mathrm{ATmC}$} & \multicolumn{3}{|c|}{ Manual } & \multirow{2}{*}{ ATmC } \\
\hline & $\min$ & \begin{tabular}{l|l|} 
max & mean \\
\end{tabular} & & $\min$ & $\max$ & mean & \\
\hline Mer & 96.66 & 99.69 & 97.23 & 99.12 & 9.58 & 99.44 & $\overline{\overline{99.58}}$ \\
\hline Ie & 98.75 & 99.62 99.15 & 98.69 & 99.25 & 99.89 & 99.72 & 99.57 \\
\hline Low Grade Glioma & 96.55 & \begin{tabular}{lll|}
99.72 & 98.85 \\
\end{tabular} & 99.16 & 99.90 & 9.94 & 99.93 & 99.91 \\
\hline
\end{tabular}

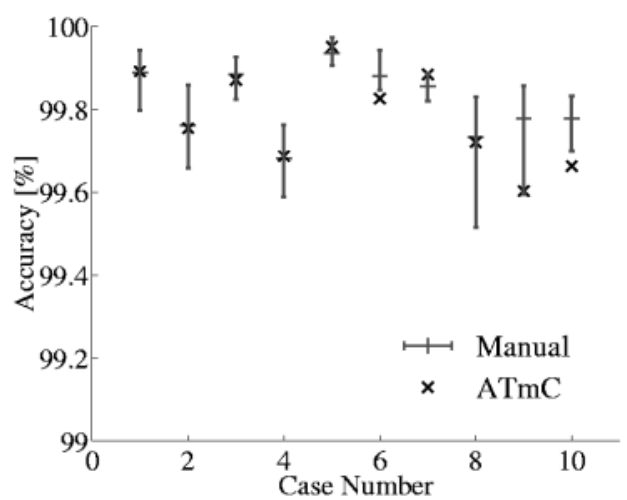

(a) Tumor

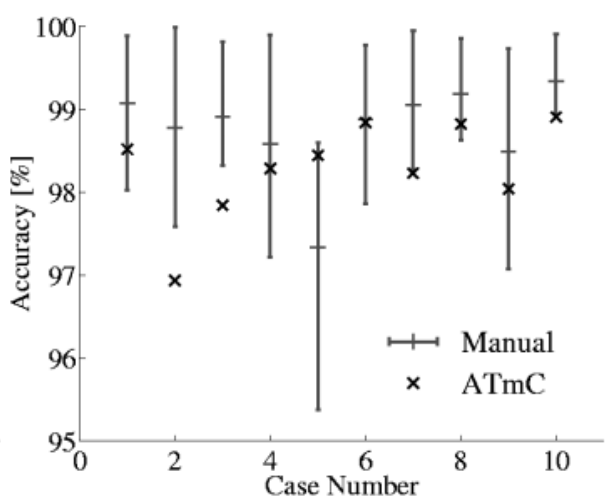

(b) Brain

Fig. 3. Segmentation accuracy of the manual (mean, minimum and maximum) and the automated method for each of the 10 brain tumor cases (1-3 Meningiomas), (7-10 Low Grade Gliomas).

The mean inter- and intra-observer variability of the automated method is lower than with manual outlining. While the inter-observer variability with the automated method is consistently lower than with the manual method, the intraobserver variability of the automated method is higher for most of the low grade glioma cases. This can be explained with the different grey value distributions of the meningioma and the low grade gliomas with respect to the brain. The meningioma tissue class partially overlaps with parts of the skin, fat in the neck and the straight and superior sagittal sinus. By restricting the region of interest (ROI) for the meningioma to the ICC, tissues that show signal intensity overlap with the meningioma are excluded and the meningioma can be successfully segmented. Low grade gliomas, however, are less distinguishable from brain tissue. Partial volume artifacts on the boundary of the brain and the tumor may cause signal intensity overlap between grey matter and tumor tissue, leading to 


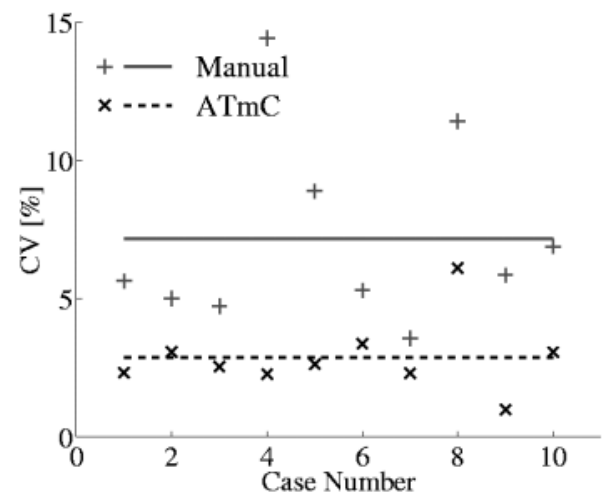

(a) Tumor

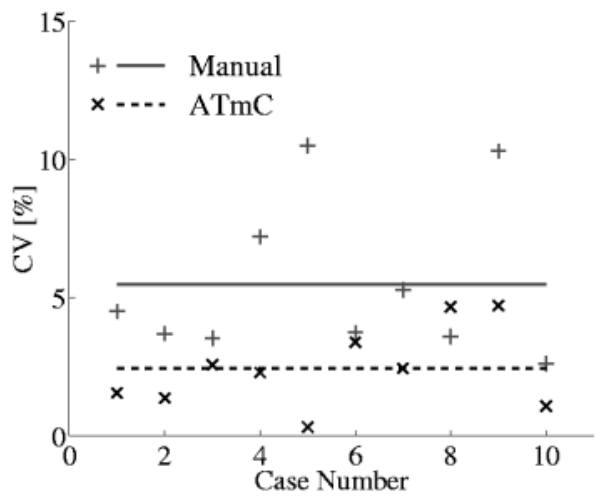

(b) Brain

Fig. 4. Inter-observer variability of manual and automated method (coefficient of variation, CV). The horizontal line marks the mean of the $\mathrm{CV}$ values.

mis-classifications, i.e. over- or under-segmentation of brain and tumor. Thus, the classifier is more sensitive to differences in the tissue prototype selection.

The mean computation time for the automated segmentation of the whole volume was 75 minutes on a Sun ES 6000 server with $20 \mathrm{CPUs}$ and $5 \mathrm{~GB}$ of RAM (Sun Microsystems, Mountain View, CA). The overall operator time was approximately. 5-10 minutes for the selection of prototypes for each of the relevant tissue classes, while manual segmentation time for a neurosurgical case has been reported to be in the range of 180 minutes [14], The reduction of operator time makes it practical to consider the integration of computerized segmentation into clinical routine.

\subsection{Conclusion and Future Work}

We have developed a method for the automated segmentation of meningiomas and low grade gliomas without edema. Accuracy and intra-observer variability of the automated method are comparable to the segmentation results from trained human observers, with improved inter-observer variability.

Further investigation is required to extend the algorithm to a broader range of brain tumors such as the glioblastoma multiforme or tumors with edema. Our algorithm is implemented on high performance computing hardware. However, through further algorithmic improvement and hardware speedups, we expect that this method will become practical in a clinical setting in the near future [13]. Currently, our tool is used in routine surgical planning to provide the basis for a clinical study based on a larger population to determine robustness and practical use in a clinical setting. 


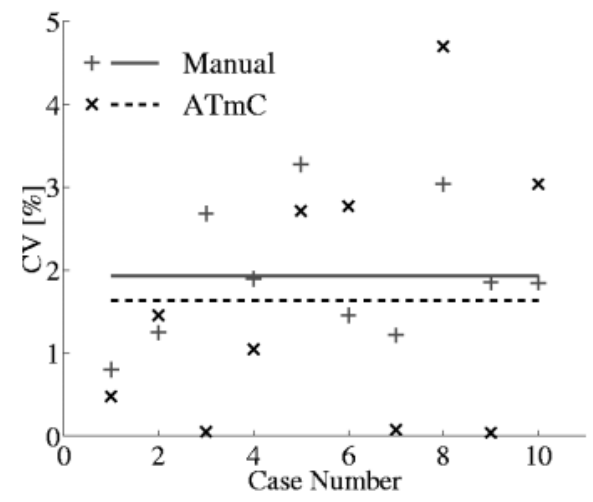

(a) Tumor

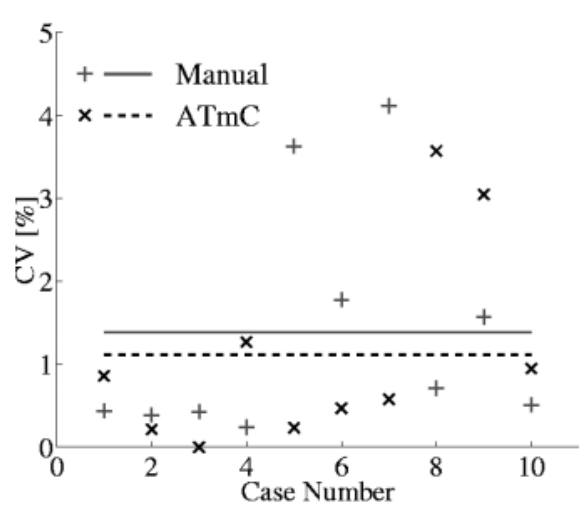

(b) Brain

Fig. 5. Intra-observer variability of manual and automated method (coefficient of variation, CV). The horizontal line marks the mean of the $\mathrm{CV}$ values.

\section{Acknowledgments}

This work was supported (in part) by a grant from the Deutscher Akademischer Austauschdienst (DAAD). This investigation was supported (in part) by a Grant from the National Multiple Sclerosis Society (SW). This work was supported (in part) by NIH grants RO1 CA 46627-08, PO1 CA67165-01A1, PO1 AG04953-14, NSF grant BES 9631710 and Darpa grant F41624-96-2-0001. The authors thank Dr. Alexandra Chabrerie, Dr. Fatma Ozlen and Dr. Daniel Boll, Brigham and Women's Hospital, Boston for their help with the manual segmentations.

\section{References}

1. PM Black, T Moriarty, E Alexander, P Stieg, and EJ Woodward. Development and implementation of intraoperative magnetic resonance imaging and its neurosurgical applications. Neurosurgery, 41:831-845, 1997. 1

2. M Clark. Knowledge Guided Processing of Magnetic Resonance Images of the Brain. PhD thesis, University of South Florida, Tampa, Florida, 1998. 2

3. DL Collins, TM Peters, W Dai, and AC Evans. Model based segmentation of individual brain structures from MRI data. SPIE Visualization in Biomedical Computing, 1808:10-23, 1992. 2

4. J Dengler and M Schmidt. The dynamic pyramid - a model for motion analysis with controlled continuity. International Journal of Pattern Analysis and Machine Intelligence, 2(2):275-286, 1987. 3

5. RO Duda and PE Hart. Pattern Classification and Scene Analysis. John Wiley and Sons, New York, 1973. 3

6. G Gerig, R Kikinis, O Kübler, and FA Jolesz. Nonlinear anisotropic filtering of MRI data. IEEE Transactions on Medical Imaging, 11(2):221-232, 1992. 4 
7. G Gerig, J Martin, R Kikinis, O Kübler, M Shenton, and FA Jolesz. Unsupervised tissue type segmentation of $3 \mathrm{D}$ dual-echo $\mathrm{MR}$ head data. Image and Vision Computing, 10(6):349-360, 1992. 2

8. P Gibbs, DL Buckley, SJ Blackband, and A Horsman. Tumor volume determination from MR images by morphological segmentation. Physics in Medicine and Biology, 41:2437-2446, 1996. 2

9. F Jolesz. Image-guided procedures and the operating room of the future. Radiology, 204:601-612, 1997. 1

10. M Just and M Thelen. Tissue characterization with T1, T2, and proton density values: Results in 160 patients with brain tumors. Radiology, 169:779-785, 1988. 2

11. M Kamber, R Shinghal, DL Collins, GS Francis, and AC Evans. Model-based 3D segmentation of multiple sclerosis lesions in magnetic resonance brain images. IEEE Transactions on Medical Imaging, 14(3):442-453, 1995. 2

12. R Kikinis, PL Gleason, TM Moriarty, MR Moore, E Alexander III, PE Stieg, M Matsumae, WE Lorensen, HE Cline, PM Black, and FA Jolesz. Computer assisted interactive three-dimensional planning for neurosurgical procedures. Neurosurgery, 38(4):640-651, $1996 . \quad 1,3$

13. R Kikinis, SK Warfield, and CF Westin. High performance computing (HPC) in medical image analysis (MIA) at the surgical planning laboratory (SPL). In Proceedings of the 3rd High Performance Computing Asia Conference 68 Exhibition, 1998. 8

14. S Nakajima, H Atsumi, A Bhalerao, FA Jolesz, R Kikinis, T Yoshimine, T Moriarty, and P Stieg. Computer-assisted surgical planning for cerebrovascular neurosurgery. Neurosurgery, 41:403-409, 1997. 2, 8

15. DW Roberts, JW Strohbehn, JF Hatch, W Murray, and H Kettenberger. A frameless stereotaxic integration of computerized tomographic imaging and the operating microscope. Journal of Neurosurgery, 65:545-549, 1986. 1

16. J Serra. Image analysis and mathematical morphology. London Academic, London, 1982. 4

17. RP Velthuizen, LP Clarke, S Phuphanich, LO Hall, AM Bensaid, JA Arrington, HM Greenberg, and ML Silbinger. Unsupervised measurement of brain tumor volume on MR images. JMRI, 5:594-605, 1995. 2

18. S Vinitski, C Gonzalez, F Mohamed, T Iwanaga, RL Knobler, K Khalili, and J Mack. Improved intracranial lesion characterization by tissue segmentation based on a 3D feature map. Magn Res Med, 37:457-469, 1997. 2

19. SK Warfield, M Kaus, F Jolesz, and R Kikinis. Adaptive template moderated spatially varying statistical classification. In Proceedings of the 1st International Conference on Medical Image Computing and Computer-Assisted Intervention, pages 431-438, Boston, MA, 1998. 2, 3

20. SK Warfield, J Dengler, J Zaers, CRG Guttmann, WM Wells, GJ Ettinger, J Hiller, and R Kikinis. Automatic identification of grey matter structures from MRI to improve the segmentation of white matter lesions. Journal of Image Guided Surgery, $1(6): 326-338,1995.2$

21. E Watanabe, T Watanabe, S Manaka, Y Mayanagi, and K Takakura. Threedimensional digitizer (neuronavigator): new equipment for computed tomographyguided stereotaxic surgery. Surgical Neurology, 27(6):543-547, 1987. 1 